\title{
Optical Fibres Integrated in Structure as a Safety Element for Pedestrians
}

\author{
Jakub Cubik ${ }^{1}$, Stanislav Kepak ${ }^{1}$, Marcel Fajkus ${ }^{1}$, Radek Marik ${ }^{2}$, Vladimir Vasinek ${ }^{1}$, \\ ${ }^{1}$ VSB - Technical university of Ostrava, Faculty of Electrical Engineering and Computer Science, Department of \\ Telecommunications \\ 17. listopadu 15, 708 33, Ostrava-Poruba, Czech Republic \\ Jakub.Cubik@vsb.cz; Stanislav.Kepak@vsb.cz; Marcel.Fajkus@vsb.cz; Vladimir.Vasinek@vsb.cz \\ ${ }^{2} \mathrm{CTU}$ Faculty of Electrical Engineering \\ Technicka 2, 166 27, Prague, Czech Republic \\ Radek.Marik@fel.cvut.cz
}

\begin{abstract}
This article discusses the use of fibre Bragg grating and Mach-Zehnder interferometer as a safety element for pedestrians. Such systems could complement existing technologies or replace them altogether. An advantage is the construction of the sensor probe without wires and metal parts along with long-distance interrogation possibility. Thanks to these features, the system is immune to electromagnetic interference, and its presence can be difficult to detect as well as affect its operation. This security system was tested on volunteers, and the results were analysed and evaluated. The first measurement was the stepping on the pad at the regular intervals. Then the system functionality has been verified with the stepping on the overlaid test pad. The last test was the ordinary walk of five volunteers over the test pad and their identification. The main task was to automatically detect all events and analyse volunteers gait in the frequency domain.
\end{abstract}

Keywords: Optical fibre, Sensors, FBG, Interferometer, Pedestrians, Security, Safety, Smart City.

\section{Introduction}

Nowadays, the emphasis is placed on improving the living situation in large cities, and the development of Smart Cities is becoming a trend. The trend implies a more intensive utilization of digital technologies in areas having a large density of population. Such a modernization helps to improve the lifestyle, health, infrastructure, awareness of people, for greater safety, to lower prices, to promote tourism and for gentle use of natural and energy resources.

Most of these metropolises are facing growing demands for property and property protection. New technologies are emerging to help safer cities. Optical fibres that can be used to construct robust and very sensitive sensory systems are becoming perspective technologies.

The creation of large urban units, the expansion of optical networks, the availability of optical fibres and components, the sensitivity of fibre optic sensors and the increasing safety demands lead to the question of using fibre optic sensors in the field of security technology. An idea is a measuring system capable of detecting and recognizing moving objects (persons) and deciding on their entry into dangerous or guarded areas. Such systems can be an add-on to existing technologies, or it is possible to used difficult detection and operate them secretly as a security technology. Other advantages of fibre optic sensors include the possibility of their connection to existing optical networks and their operation over long distances. Therefore, fibre optic sensors and their use are the future of the security sector.

The principle of guiding light by reflection was first demonstrated in 1840, and since then enormous progress has been made in the field of fibre optics. Nowadays, the use of light for high-speed data transmission in backbone networks is ubiquitous, and in the near future it is envisaged that everyone will have access to optical fibre-based connectivity at home (so-called FTTH fiber-to-the-home). The field of fibre-based optical sensors has been developing alongside progress in the development of fibre optics for data transmission although at a slower pace. However, at present, there is a great interest in this field primarily due to the advantages of high sensitivity over long distances, the dielectric nature of optical fibre providing immunity to electromagnetic interference and low signal attenuation over long distances $[1,2]$. 
This publication builds on previous research undertaken by our research team [3 - 8]. In this paper, this research is extended to demonstrate previously unknown facts about the behaviour of fibre-optic interferometric sensors.

There is a small number of security systems which use FBG or interferometer as a sensing element. Majority of research teams deal with one of these methods, but in this paper, we would like to proof the benefits of using both sensing methods together [9].

\section{Experimental setup}

For purposes of detection and monitoring of key walk markers, a test pad of $400 \times 200 \times 2 \mathrm{~mm}$ has been created. The pad is made up of a polydimethylsiloxane polymer in which the Bragg grating is embedded in a tube and the Mach-Zehnder interferometer measuring arm with the length of $3 \mathrm{~m}$. The diagram of the pad is shown in Figure 1.

To achieve the sensitivity of Bragg grating across the entire length/surface of the pad, the optical fibre with FBG was insert into a tube with an inner/outer diameter of $0.5 \mathrm{~mm} / 0.9 \mathrm{~mm}$. The tube had a length of $370 \mathrm{~mm}$, the fibre with FBG was fixed at the ends in the PDMS to achieve a Long-gauge type of sensor that measures deformations along the length of the tube.



Fig. 1: Scheme of the PDMS test pad with the Bragg grating and arm of the interferometer.

\subsection{Cover layers}

The measuring pad made of PDMS is made according to diagram 1. A yellow thread is embedded around the circumference, representing the M-Z interferometer measuring arm. Longitudinally, there is an optical fibre with FBG in the tube. A part of this experiment was the use of cover layers to serve as a pad protection. The aim of the measurement is therefore the analysis of the use of various cover layers and their influence on the measurements. Linoleum, acrylic glass and rubber strip were used as cover layers. The dimensions of the cover layers were $340 \times 180 \times 2 \mathrm{~mm}$.

\section{Measurement and results}

The measurement was divided into two parts. The first part is designed to test the functionality of the proposed measuring assembly. The second part is the measurement of controlled passages, determination of their validity and frequency analysis.

\subsection{Verification of functionality}

The verification of functionality was performed simultaneously with the FBG evaluation unit and the M-Z interferometer evaluation unit. The initial measurement was performed by controlled repeated steps of 2 people on the PDMS pad without and with different cover layer variants. The following figures show the courses of controlled steps of the first person with different cover layers. 


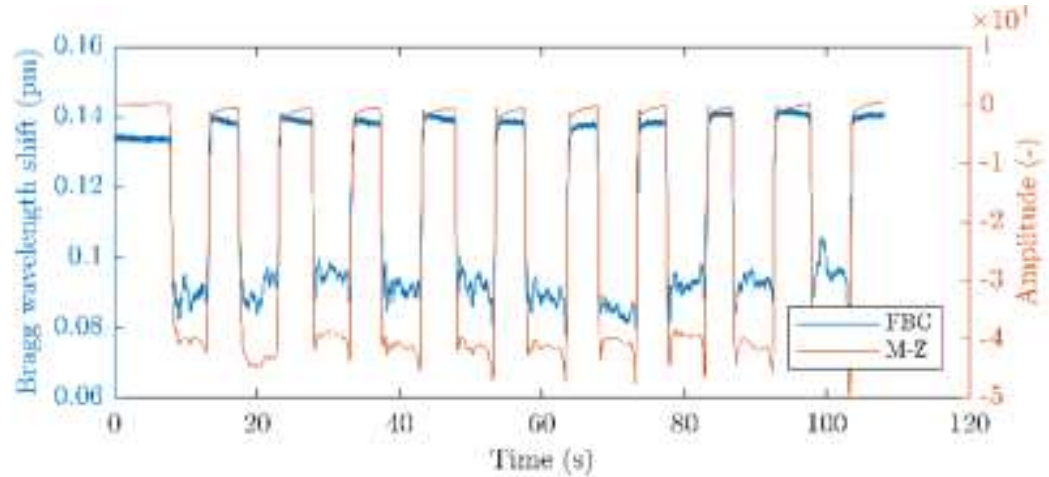

(a)



(b)

Fig. 2: Measured signal of controlled steps (person \#1); (a) PDMS only; (b) the linoleum liner.

Initially, the controlled steps were performed at an interval of $5 \mathrm{~s}$ ( $5 \mathrm{~s}$ step, $5 \mathrm{~s}$ release). This experiment was used to verify the functions of individual systems. After that, fast stepping, which is more like a quick walk, was also tested. The result shows that the FBG sensor and interferometric sensor in the measuring pad allow for fast step-on detection without significant signal degradation due to the relaxation of PDMS flexible material so that individual fast steps are distinguishable (no single step signals are merge). This verification is shown in Figure 3.



Fig. 3: Rapid stepping (27 steps in $15 \mathrm{~s}$ ). 


\subsection{Event detection}

The input signal has an almost stable noise component. The step events can be treated as nonstationary impulses detected as anomalies deviating from the noise pattern. Furthermore, the input signal exhibits a nonlinear trend component that cannot be neglected.

Our event detection processing chain is a modification of the approach based on extreme value theory proposed in [10 The approach is built around the asymptotic distributions of extrema values [13]. It uses both traditional extreme value methods, i.e. one based on the block maxima distribution and the other using the peak over threshold (POT) distribution. The POT method combined with declustering (a single value for each sequence of values exceeding the threshold) is suitable for non-stationary signals. Given reasonable assumptions, the asymptotic extrema value distribution analytical form can be derived and shown to have three families, Frechet, Gumbel, and Weibull. It is assumed in our detection schema that the extreme value distributions computed along the signal belong to the Weibull's family that is constrained by its limit that is treated as the threshold base level. The percentiles can be selected in such a way that the limit estimation is very robust against distribution outliers. Thus, the approach capable to estimate efficiently and robustly the extreme values is able to find the range of a noise component. Everything, except for the noise, is detected as an anomaly and then stored as an event. The actual thresholding is performed as hysteresis thresholding operating with two thresholds derived from the threshold base level and the distance of the percentiles. The higher threshold signals an event, while the lower threshold is used for event period identification.

First, the range of noise component is delimited. The envelopes can be constructed for both stationary and non-stationary signals. The outlier score is computed based on extreme value envelopes. The difference suppresses significantly the trend component. However, it is also sensitive to rapid signal amplitude changes that are related to step events. The outlier score is again processed with the aim to identify the maximum level of its noise component. It is assumed that the range of the noise component can change with regard to a processing window. Block extreme value distributions are estimated using the sliding window, while POT distributions are estimated using a buffer. As anomaly events might be quite dense, the range of the outlier score noise component is identified in several steps. In each step, detected possible anomalies are avoided and suppressed.

\subsection{Measurement and analysis}

The measurements were carried out on the above-mentioned pad and we specifically focused on data from the FBG sensor. Five volunteers aged 25-35 were selected. They made more than 100 steps on the pad in one walk direction and without footwear. The task of measuring and subsequent analyses included the detection of individual steps, determination of validity of these steps and evaluation in the spectral area. Measurements were carried out with a focus on maintaining constant conditions (step length, transition speed, and temperature). 


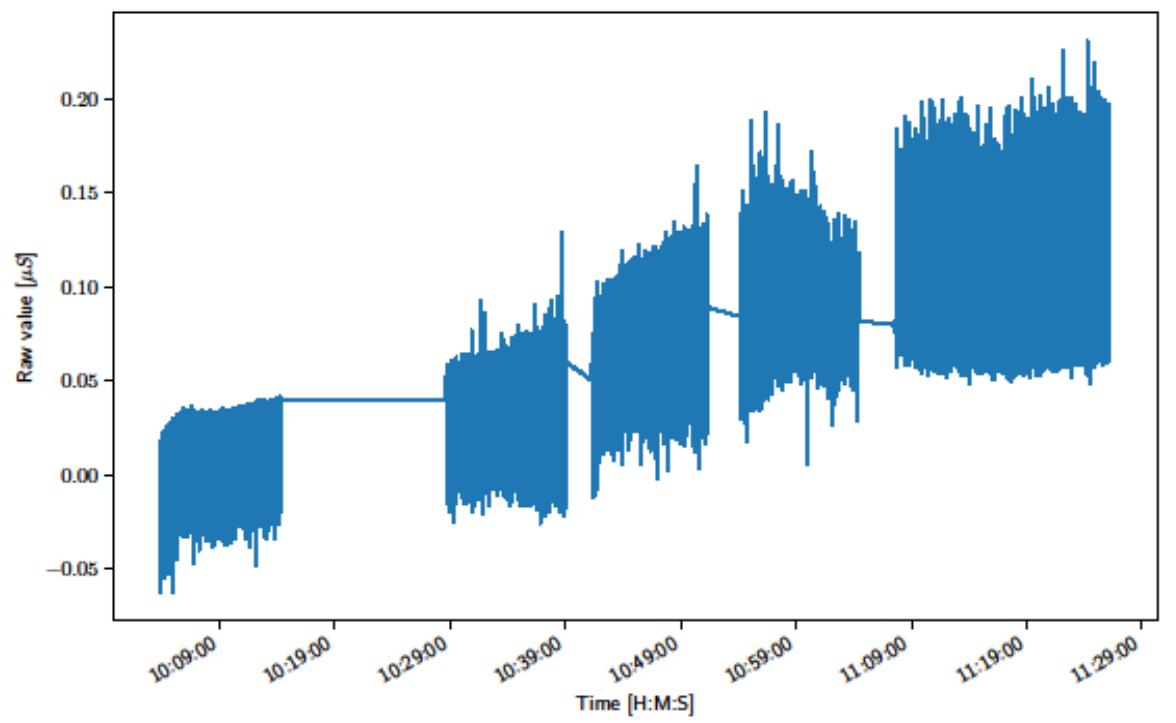

Fig. 4: Measured signal of controlled steps (5 persons).

Figure 4 shows the entire unprocessed measurement signal. Subsequently, an event detection algorithm, see Section 3.2, was applied to the signal. Thus, 548 events corresponding to individual steps were found in the signal.

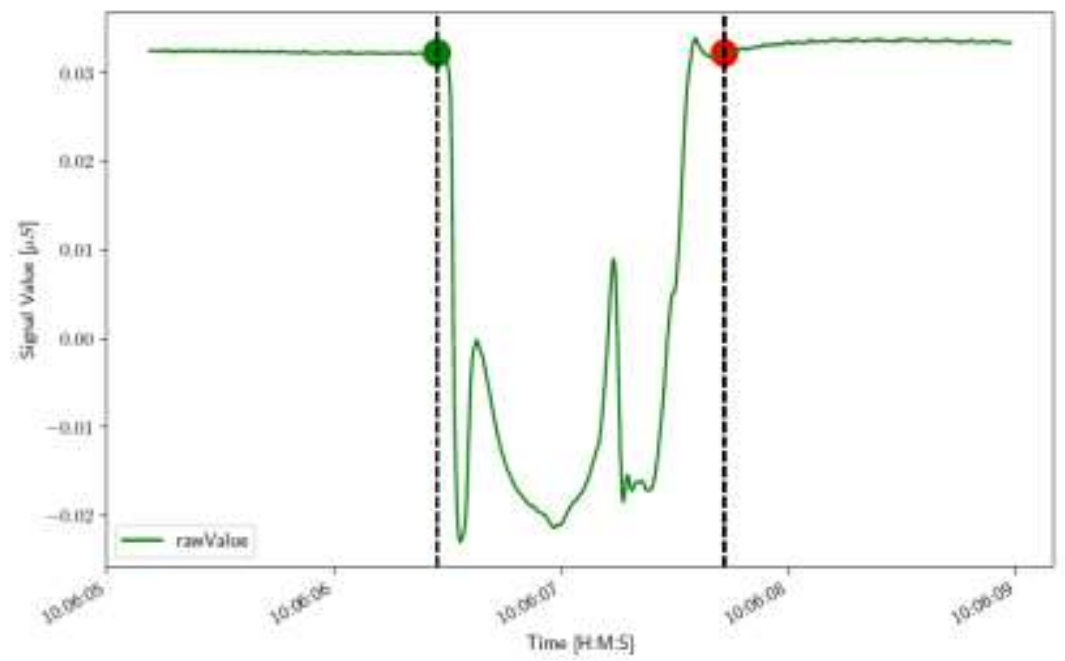

Fig. 5: Signal of first person (events 20). 


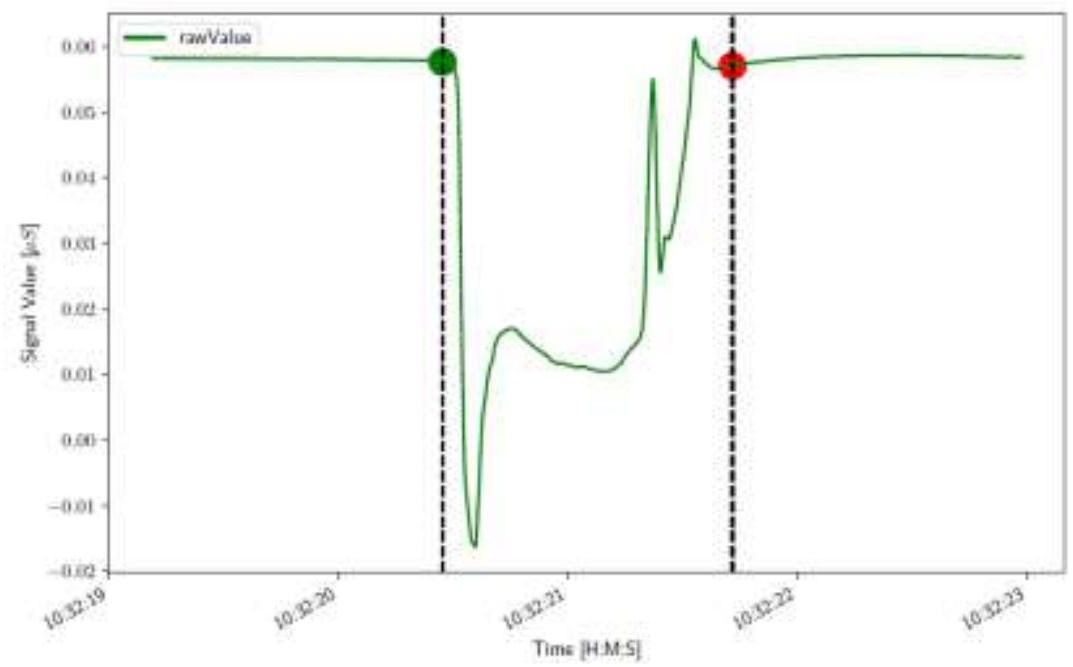

Fig. 6: Signal of second person (events 146).

Figures 5 and 6 show a single event signal. These events were randomly selected to demonstrate the difference between individual steps (people). The course of the step on the pad differs at first glance, which can lead to the identification of essential information for identifying a particular person in subsequent spectral analysis.

It was also found that the event search algorithm after manual pass reached the validity of $98.72 \%$. The system was able to find all the individual steps but also detected seven false events $(105,211,315,316,434,507,546)$. These events, according to the algorithm settings, should not be recorded and this error will be subjected to further analysis. An example of such records is shown in Figure 7.

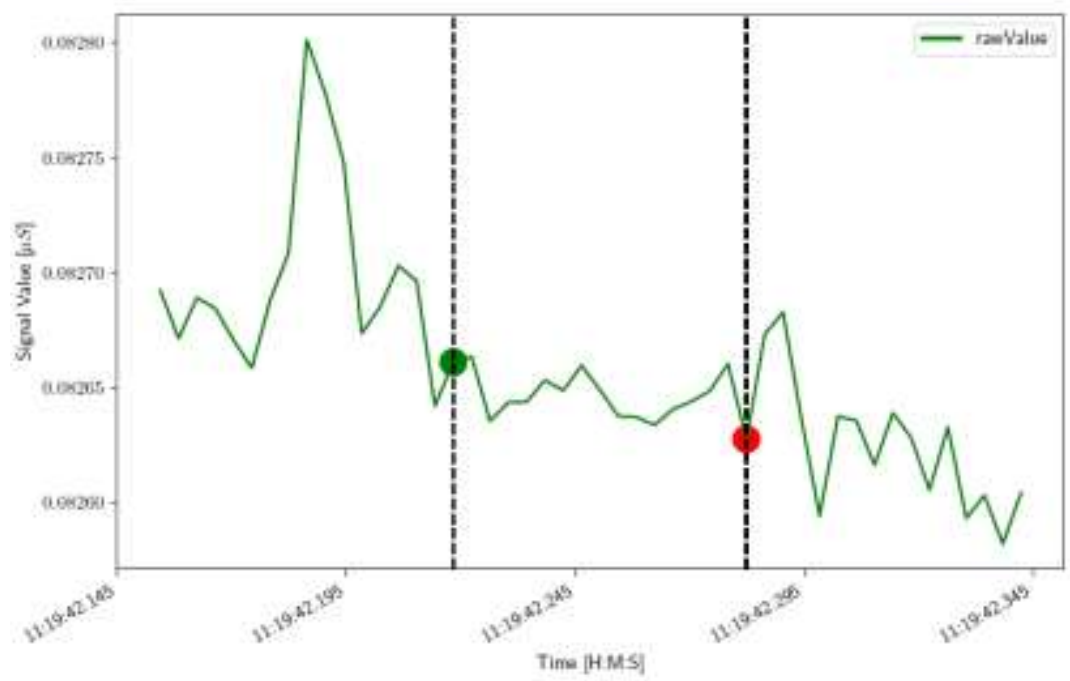

Fig. 7: Examples of mistakenly recorded events (507). 


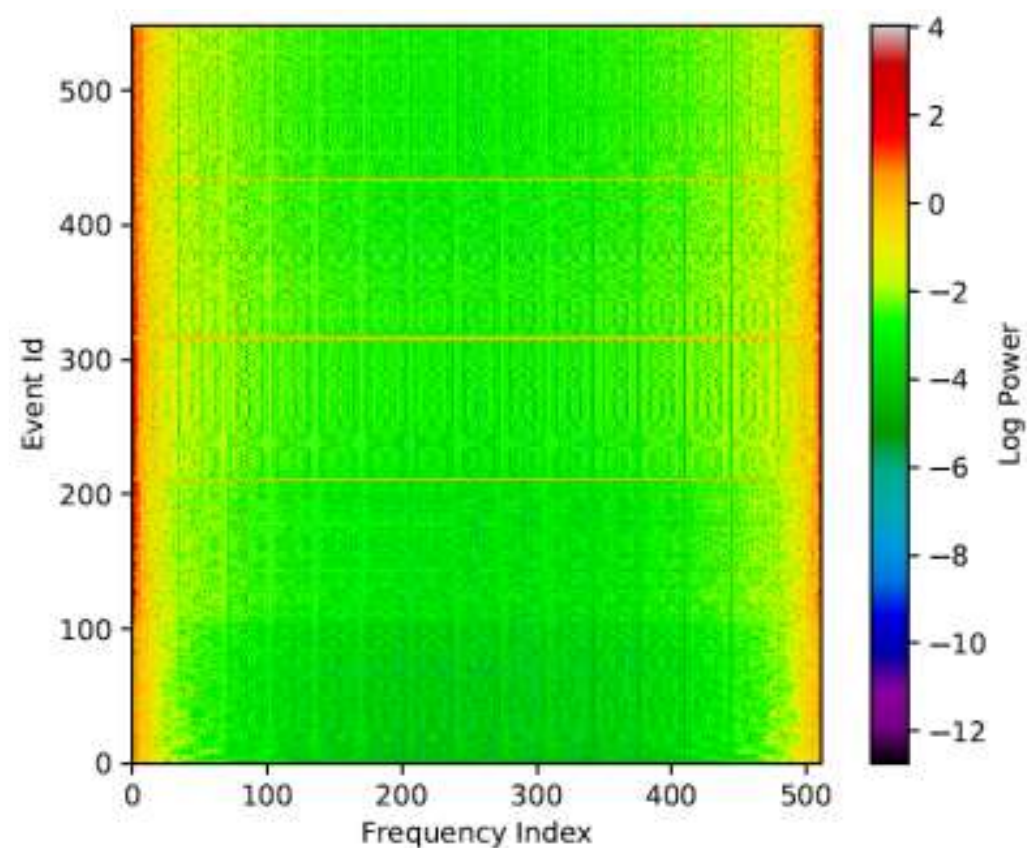

Fig. 8: Overview of frequency responses of individual persons. The individuals are separated by transition events exhibiting an anomaly frequency spectrum.

In order to demonstrate the frequency response of each person's steps, a procedure was chosen whereby each event was added to 512 samples (by adding the last known value), a fast Fourier transformation was performed and the energy of the resulting samples was inserted by columns into a matrix (each column represented a frequency of one event). The matrix is displayed as a heat map (spectrogram). Changes were manifested among users especially at low frequencies (we worked with mechanical vibrations).

The corresponding frequency overview is shown in Figure 8. The frequency energy is shown in the logarithmic scale. As presumed, different users' steps can be distinguished. Individual users are separated by one transition event.

\section{Conclusion}

The results of controlled test steps should prove that both types of sensors are capable of detecting individual steps. This was confirmed both for the FBG, and for the interferometric system. The FBG sensor had a lower response rate of about 10 $\mu \mathrm{m}$. Testing also proved that the FBG sensor is sensitive to pressure at all locations of the pad.

Another part is the evaluation of controlled passage on the pad. The range of the measured people focuses on more (100 or more) steps of one person with a focus on maintaining constant conditions. The system analyzed 548 events, with 7 false events reaching $98.72 \%$ (the number of relevant events/total number of events). The frequency spectrum response analysis shows that the system should be able to distinguish a change of an event. This result opens a space for an application of more sophisticated techniques of pattern recognition and classification, e.g. based on recent advances in deep learning methods. In the case of walk identification, it should be a mechanism that will be able to learn and evaluate changes based on the defined parameters. These parameters and identification of walk changes should be created in co-operation with a specialized (probably medicinal) defined reference device.

The introduced identification system, complemented by event processing software and learning (adaptive) algorithm, can find its application in security technology, transport, or medicine. 


\section{Acknowledgements}

This paper was supported by the Ministry of the Interior the Czech Republic project No. VI20152020008 Complex security of critical infrastructures and objects solved with fiber optical sensors with usage of modern systems. The research described in this article could also be carried out thanks to the active support of the project No. SP2019/80. This research has been partially supported by the Ministry of Education, Youth and Sports of the Czech through the grant project No. CZ.1.07/2.3.00/20.0217 within the frame of the operation program Education for Competitiveness financed by the European Structural Funds and from the state budget of the Czech Republic.

\section{References}

[1] D. Krohn, "Fiber optic sensors: fundamentals and applications," Instrument Society of America, Research Triangle Park, NC, 1992.

[2] J. M. López-Higuera, Handbook of Optical Fibre Sensing Technology. New York: J. Wiley and Sons, 2002, pp. 227245.

[3] J. Cubik, S. Kepak, J. Doricak, V. Vasinek, J. Jaros, A. Liner, M. Papes, and M. Fajkus, "The Usability Analysis of Different Standard Single-Mode Optical Fibers and its Installation Methods for the Interferometric Measurements." Advances in Electrical and Electronic Engineering, vol. 11, no. 6, pp. 535-542, 2013.

[4] V. Vasinek, J. Cubik, S. Kepak, J. Doricak, J. Latal, and P. Koudelka, "Mach-zehnder interferometer for movement monitoring," in the Proceedings of SPIE - the International Society for Optical Engineering, vol. 8370, 2012.

[5] J. Cubik, S. Kepak, J. Doricak, V. Vasinek, A. Líner, and M. Papes, "Interferometric sensor based on the polarizationmaintaining fibers," in the Proceedings of Czech-Polish-Slovak Optical Conference, 2012.

[6] J. Cubik, S. Kepak, M. Fajkus, O. Zboril, J. Nedoma, and V. Vasinek, "Fixing methods for the use of optical fibers in interferometric arrangements," in the Proceedings of SPIE - the International Society for Optical Engineering, vol. 10142, 2016.

[7] M. Fajkus, J. Nedoma, P. Siska, L. Bednarek, S. Zabka, and V. Vasinek, "Perimeter system based on a combination of a Mach-Zehnder interferometer and the bragg gratings," Advances in Electrical and Electronic Engineering, vol. 14, no. 3, pp. 318-324, 2016.

[8] J. Nedoma, M. Fajkus, R. Martinek, M. Novak, O. Zboril, J. Jargus, K. Witas, F. Perecar, and V. Vasinek, "Influence of different encapsulation types and shapes of polydimethylsiloxane on the temperature sensitivity of the FBG," in Proceedings of SPIE - The International Society for Optical Engineering, 2017.

[9] J. Jiang, Y. Jiang, D. Liu, S. Wang, and X. Li, "Fiber-optic perimeter security system based on dual Mach-Zehnder interferometer structure," Guangxue Jishu/Optical Technique, vol. 41, no. 3, pp. 193-196, 2015.

[10] R. Marik, "Threshold Selection Based on Extreme Value Theory," in Březina, T. \&amp; Jabłonski, R. (Eds), Mechatronics 2017: Recent Technological and Scientific Advances, Springer International Publishing, 2017, pp. 529537.

[11] R. Marik, R., "Thresholding Using Extreme Value Theory Threshold Models," in Maga, D., Stefek, A., Brezina, T. (Eds), Proceedings of the 2018 18th International Conference on Mechatronics - Mechatronika (ME) Brno, Czech Republic, Brno University of Technology, 2018, pp. 434-441.

[12] R. Marik, L. Bohac, "Non-stationary Events Detection Based on Extrema Value Theory," in the 2018 5th International Conference on Systems and Informatics (ICSAI 2018), 2018, pp. 859-864.

[13] S. Coles, An introduction to statistical modeling of extreme values. Springer, 2001. 